\title{
Effect of Target Coating Thickness on the Abrasive Waterjet Machining Response of 8YSZ Segmented Thermal Barrier Coatings
}

\author{
R. Patgunarajah ${ }^{1} \cdot$ J. P. Borrmann ${ }^{1} \cdot$ J. E. Döring ${ }^{1 *} \cdot$ A. Sharma ${ }^{2}$ \\ 1 Siemens Energy Global GmbH \& Co. KG, Berlin (Nonnendamm)/ Aachen / Mülheim an der Ruhr, Germany. \\ ${ }^{2}$ Siemens Energy Inc., Charlotte, NC, USA.
}

\begin{abstract}
Segmented thermal barrier coatings (STBC) are a more strain-tolerant and erosion resistant alternative of the conventional porous thermal barrier coatings for hot gas components in gas turbines. Due to their relatively higher thermal conductivity and the ever-increasing turbine inlet temperature, there is a need for thicker STBCs. This paper presents an investigation of the microstructures and properties of STBCs with respect to coating thicknesses. Coatings with two significantly different thicknesses were fabricated under identical conditions and evaluated using standard metallographic methods. It was found that the microstructures for thin and thick coatings had subtle differences and hardness values decreased with in increasing thickness. When subjected to an abrasive waterjet machining process, the thicker STBCs showed a higher wear rate. A hypothesis is proposed to explain the observed thickness dependent changes and the microstructures and properties of STBCs.
\end{abstract}

CIndian Thermal Spray Society and INScienceIN. 2020. All rights reserved
ARTICLE HISTORY

Received 01-12-2020

Revised 10-12-2020

Accepted 11-12-2020

Published 17-12-2020

\section{KEYWORDS}

Thermal Barrier Coatings Segmentation Cracks

Gas Turbine

Thermal Spraying

Abrasive Waterjet

Machining

\section{Introduction}

The specific power output and the efficiency of a gas turbine engine are directly related to the turbine inlet temperatures (TIT). To reduce fuel consumption and its environmental impact, there is a constant drive to increase the TIT. As a result, thermal barrier coatings (TBCs) have become an integral part of hot gas components of a gas turbine engine and are being continuously developed to improve robustness and reliability [1-4].

There are two primary TBC microstructures sprayed by conventional (powder-based) air plasma spray (APS) processes - (1) Porous and (2) Dense-segmented. Porous TBCs are in general characterized by the presence of globular voids of various sizes, shapes and orientations, interlamellar boundaries, and microcracks [5-6]. The presence of pores and interlamellar boundaries renders the thermal conductivity of the coating lower than its bulk counterpart, thereby providing the thermal barrier function in the turbine engine. The presence of pores and microcracks also imparts a certain degree of strain tolerance to the porous TBC which helps them endure the thermal cycling in the engines. However, such microstructures generally have poor long-term thermomechanical durability due to sintering induced closure of small pores and cracks and associated changes in coating properties [7].

Dense-Segmented Thermal Barrier Coatings (STBCs) on the other hand, are characterized by dense coating microstructure with periodic macrocracks perpendicular to the coating/substrate interface (vertical macrocracks). Due to the absence of pores and macrocracks being oriented parallel to the direction of heat-flow in the turbine engines, the thermal conductivity of STBCs is higher than that of the porous TBCs. However, due to their higher facture toughness and the greater strain tolerance imparted by the vertical macrocracks, SBTCs show better thermal cyclic performance. Segmented coatings are also more resistant to sintering during thermal exposure because the coating segments between the macrocracks are already dense assprayed.

STBC was originally developed and patented by Thomas Taylor [8] more than 30 years ago. In his 1989 patent, he provided the STBC microstructure formation mechanism and emphasized the role of a temperature gradient between well-bonded splats with a higher temperature at the coating surface. Since then there have been several investigations to understand the formation mechanism of STBC and the role of coating processing parameters [1, 913]. The current understanding of the formation of STBCs is as follows: Upon cooling and solidification, the hotter splats would shrink more than the underlying splats and thus form vertical cracks through biaxial tensile stresses within the individual splats. When the coating is deposited at low substrate temperatures, the micro cracking together with the globular pores and relatively weaker inter-splat bonding helps relieve the deposition stress thereby inhibiting the formation of macroscopic segmentations. This leads to the formation of porous TBCs. However, when the coating is deposited on a higher surface temperature (i.e., increased inter-splat bonding) with spraying conditions supporting a dense microstructure, the stress buildup in coating with increasing thickness, has no relief mechanism. The stress/strain buildup in the coating can only be sustained up to a point, after which the system incorporates segmentation cracks to maintain stability.

Accordingly, the methodology to create STBC microstructure (i.e., controllably creating segmentation during spraying) in our present work involved deposition of dense coating on a hot surface (with surface temperature preferably higher than the critical bonding temperature 

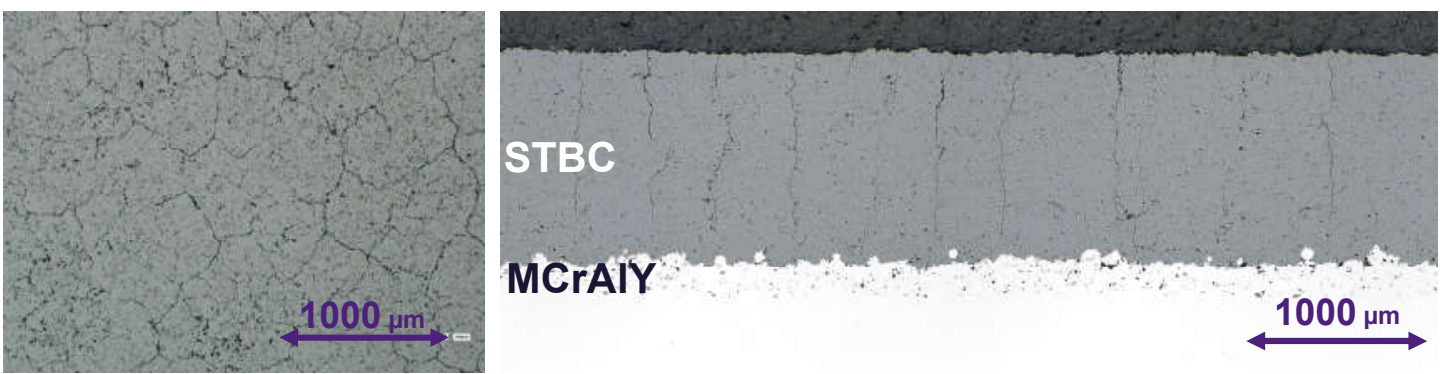

Figure 1: Coating structure with a high level of vertical cracks - left: Top surface view; right: microsection

[9], and maintaining suitable thermal gradient through the thickness of the deposit. The typical architecture of STBCs shown in Fig. 1. The macrocracks between segments open with tensile loadings and close with compressive loadings. The increased stress tolerance of STBCs helps extend their cyclic life behavior and denser microstructure improves the erosion resistance compared to conventional porous coatings. $[1,2,14]$

For a given thermal conductivity, TBC thermal resistance is proportional to its thickness. In serial production settings, design required target thickness for a given component is achieved by first determining the application rate (thickness/pass) for a fixed set of spray parameters and then applying the required number of spray passes to achieve the target thickness. For a fixed set of spray parameters, coating microstructure and properties are generally believed to be independent of its thickness.

For the case of STBCs, however, Shinde et al. [1], showed that coating remains free of vertical cracks up to $200 \mu \mathrm{m}$ thickness, at which point vertical segmentations develop in the coating. From this point on vertical cracks are generated in the coating and the crack density (number of vertical cracks per unit length) remains practically independent of coating thickness. These authors also showed that the elastic modulus of the STBCs measured by ex-situ bi-layer curvature methods $[3,15]$ decreased continuously with increasing coating thickness. They explained this decrease in the elastic modulus to be a result of increasing ratio of the cracked to uncracked portions. The paper also showed that the in-situ deposition stress in STBCs continuously decreased with thickness after the onset of vertical segmentations. However, they do not elucidate this effect in any detail. Nevertheless, the paper highlighted that certain properties of STBCs may have a thickness dependence.

In our current work, we noted that the response of the STBCs are indeed different for thin and thick coatings when post processed using abrasive waterjet machining. A systematic investigation of this behavior led to the discovery of subtle changes in the coating microstructure as a function of its thickness. This paper describes the coating fabrication and abrasive waterjet machining processes applied in this work and attempts to explain the observed thickness dependence of coating properties. The significance of this work is that unlike conventional belief, it is not always appropriate to assume thickness independence of coating properties.

\section{Experimental}

\section{Coating fabrication via Thermal Spraying}

Stainless-steel plates (X5CrNi18-10) of dimensions $100 \mathrm{~mm} \times 80 \mathrm{~mm} \times 10 \mathrm{~mm}$ were coated with a duplex system of MCrAlY (M - Metal (Ni, Co, Fe or a combination thereof), Cr-Chrome, Al - Aluminum, Y - Yttrium) bondcoat. First, high velocity oxygen fuel spraying technique (HVOF) was used to apply the fine MCrAlY powder to produce the dense bond-coat layer which provides oxidation protection. It was followed by the application of coarser powder by atmospheric plasma spraying (APS) to form a relatively thin and rough layer to ensure an optimized bonding with the ceramic layer. The samples were then placed in the furnace for diffusion annealing.

Next, the ceramic layer was applied using the F4-MB plasma torch (Oerlikon Metco, Westbury NY USA) with a fixed nozzle diameter and a double injection spray spot setup. The ceramic feedstock used for the TBC was $7-8 \%$ yttria partially stabilized zirconia powder (Amperit 825, Höganäs $\mathrm{GmbH}$, Germany) was produced by a fused and crushed powder manufacturing process. The powder particles have angular, blocky morphology and a prespecified particle size distribution per powder supplier's specifications. The coating parameters used to produce STBC in this work are provided in Table 1.

Table 1: Spraying parameters used to obtain STBC coatings

\begin{tabular}{l|l}
\hline \multicolumn{1}{c|}{ Attribute } & \multicolumn{1}{c}{ Value } \\
\hline Spray Process & APS \\
Spray torch & F4MB \\
Powder Composition & $6-8$ wt\% YSZ \\
Substrate Type & Stainless-steel \\
Gun power (kW) & $\sim 40$ \\
Primary gas & Argon \\
Secondary gas & Hydrogen \\
Primary / Secondary flow & $\sim 4: 1$ \\
ratio & \\
Carrier Gas Type & Argon \\
Carrier Flow (NLPM) & Proprietary \\
Powder Feed Rate (g/min) & Proprietary \\
Spray Distance (mm) & $<120$ \\
\hline
\end{tabular}

The coating routine had a fixed step size and a constant offset for every second pass. The offset depends on the geometry of the spray spot to distribute the locally supplied temperature over the layers, thereby enabling a homogeneous layer structure and preventing delamination. The specimens had a fixed spray distance from the nozzle exit. The same applies to the injection distance about the center of the nozzle cross section. At a pre-investigated constant input current and an approximate 4:1 argonhydrogen ratio of the primary and secondary operating gases, the power values shown in Fig. 2, were recorded. Minimal power fluctuations of less than $5 \%$ of the measured values were observed. Similarly, a stable value of gun voltage (not presented) was also observed as a 
function of the electrode lifetime. These measurements indicate a very stable coating process in relation to the operating hours during which the coatings samples of this study were sprayed.

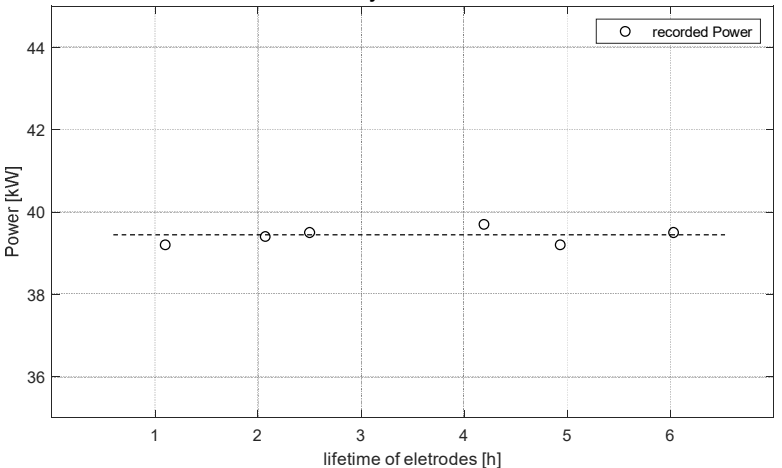

Figure 2: Torch power as a function of the lifetime of electrodes

Two sets of different coating thicknesses (in the following referred to as "thin" and "thick") were produced under identical spray parameters, where the coating thickness was varied via adjustment of the repetitions of the meandering robot program. The cathode and anode pair were run on defined calibration parameters before the start of coating. This procedure prevented a restrike frequency drop of the electrodes over the coating time and ensured high enthalpy stability of the plume. To maintain an adequate surface temperature, the surfaces to be coated were preheated to enhance splat-to-splat bonding and to reduce splat fragmentation [1]

\section{Abrasive Waterjet Machining of Coatings}

The practical abrasive waterjet machining investigations were accomplished to a customized 5-axis waterjet machine (H.G. Ridder Automatisierungs $\mathrm{GmbH}$, Hamm, Germany) based on Type WARICUT micro-max. This machine is especially customized for a flexible waterjet machining of complex free-form surfaces like on gas turbine components. The linear XYZ-axes including the machining head are designed in gantry construction. Additionally, there is a turn swivel table including the workpiece fixture system installed in the machine bed. For the machining head itself a typical abrasive waterjet head with injector technology also by H.G. Ridder with a 0.12 $\mathrm{mm}$ water orifice in combination with a $0.54 \mathrm{~mm}$ focusing tube was utilized. A garnet sand with granulation 200 Mesh (Particle size: $75 \mu \mathrm{m}$ ) was used as abrasive media. A picture of the waterjet machine is shown in Fig. 3.

For waterjet machining of the coated STBC samples, the tool path was a meander-form with a hatch distance of 0.27 $\mathrm{mm}$, which is half of the focusing tube diameter. This leads to a mostly plane notch root with low waviness. The machining angle was set to $90^{\circ}$, machining distance to 4 $\mathrm{mm}$ and feed rate to $1625 \mathrm{~mm} / \mathrm{min}$. Regarding water pressure $p$ and abrasive mass flow $\dot{m}_{A}$ the following two combinations were tested for both the thin and thick STBCs:

Combination 1: $p_{1}=1300 \mathrm{bar}, \dot{m}_{A 1}=12 \mathrm{~g} / \mathrm{min}$.

Combination $2: p_{2}=1800 \mathrm{bar}, \dot{m}_{A 2}=20 \mathrm{~g} / \mathrm{min}$

A schematic overview is shown in Fig. 4 . For comparing the waterjet machining response of thin and thick TBCs, all waterjet machining parameters were kept constant for a given set of samples.
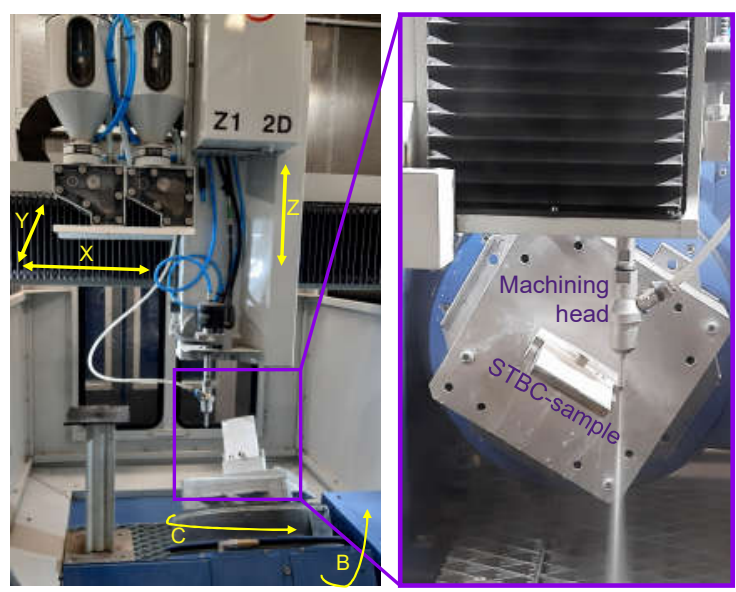

Figure 3: Customized 5-axis waterjet machine

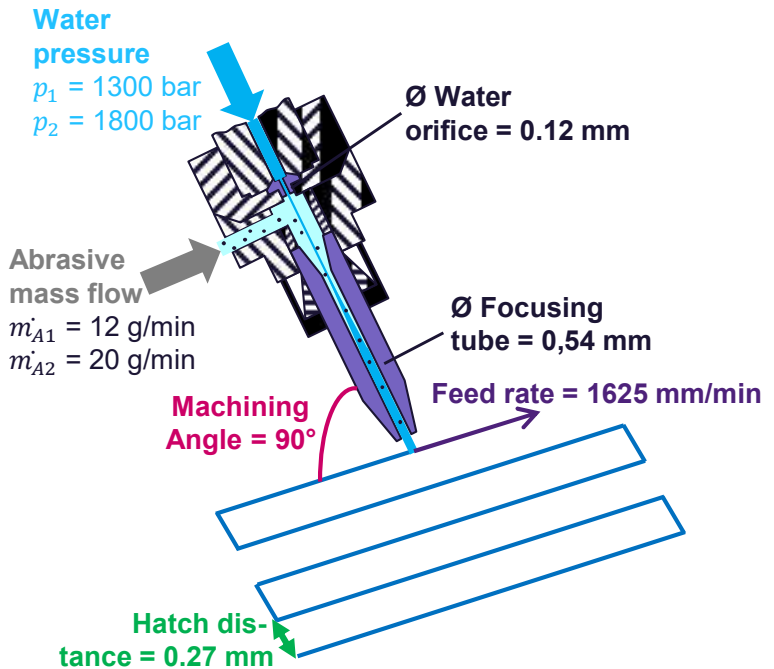

Figure 4: Schematic overview of waterjet head configuration and used parameters

\section{Experimental Approach}

The experimental approach consisted of processing of STBCs using two manufacturing technologies - thermal spraying and abrasive waterjet machining. Furthermore, different types of analysis of post procedures were conducted. In a first step, the STBC has been applied on substrate plates with two different coating thicknesses. The specimens were weighed before and after coating to determine deposit efficiencies. After the coating of the samples, the thicknesses of the coatings were determined by eddy current measurement and metallographic analysis. Measured thickness was used to determine the application rate (or deposition rate) in terms of thickness per pass. In the next step, the specimens were metallographically prepared and examined using an optical microscope. In the metallographic analysis, the STBC microstructures were analyzed in different portions of the cross sections. Additionally, the Rockwell macrohardness (HR15N and HRC) and Vickers micro hardness (HV) were measured by a Zwick Roell universal hardness measurement device. A diamond cone with a point angle of 120 degree was used for the Rockwell hardness measurement of the as-sprayed 


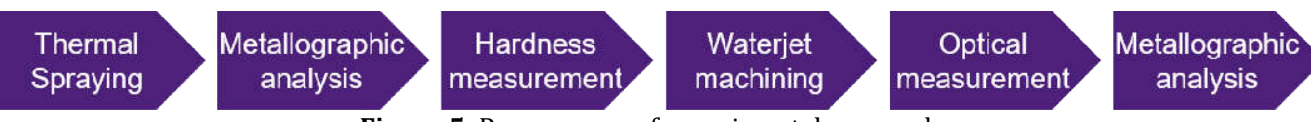

Figure 5: Process map of experimental approach

samples. The measurement procedure was calibrated to the unmachined ceramic surface of the samples. The HR15N and HRC macro hardness tests were implemented in accordance with EN ISO 6508-1 and in compliance with the defined application and holding times. The microhardness measurements were executed on the polished microsections such that the location of the diamond pyramid indentation was in middle of the total coating thickness. Afterwards the waterjet machining process was performed with two different parameter combinations (combinations 1 and 2 described earlier). Each combination was tested five times on the thin and thick STBC material. The notch geometries and surfaces were measured and compared using an optical 3D surface measurement system (Alicona Type Infinite Focus, Alicona Imaging GmbH, Graz, Austria). In addition, the microsections were compared to the as sprayed ones. The overall experimental approach is summarized in Fig. 5 in form of a process map.

\section{Results and Discussion}

The coating deposition efficiency and the coating application rate of the samples calculated from the mass and thickness gain, respectively are shown in Fig. 6 . These characteristics show a scatter of less than $5 \%$, revealing a high process stability during the entire experimental test campaign. This implies that there was no obvious difference in the spray characteristics between the thin and thick coatings.

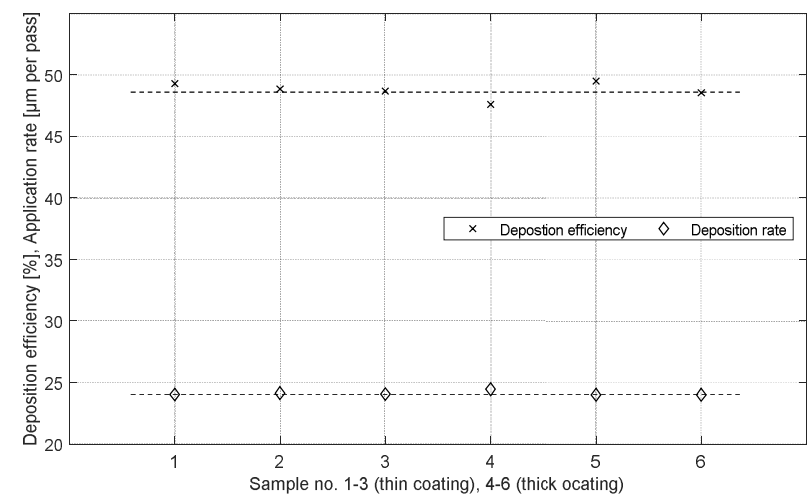

Figure 6: Deposition efficiency and application rate in dependence of coating thickness

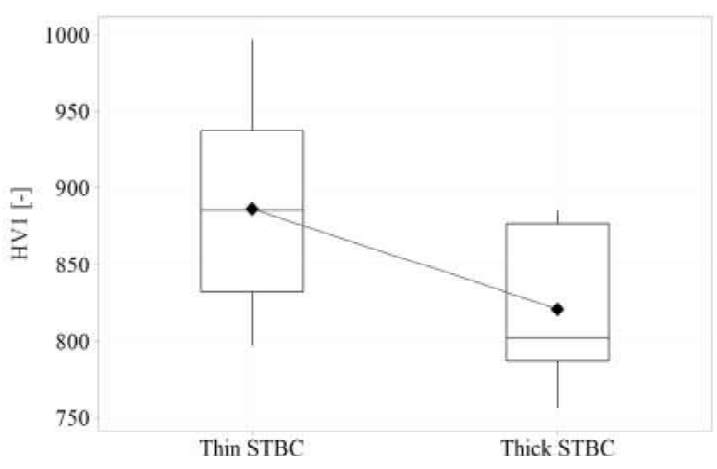

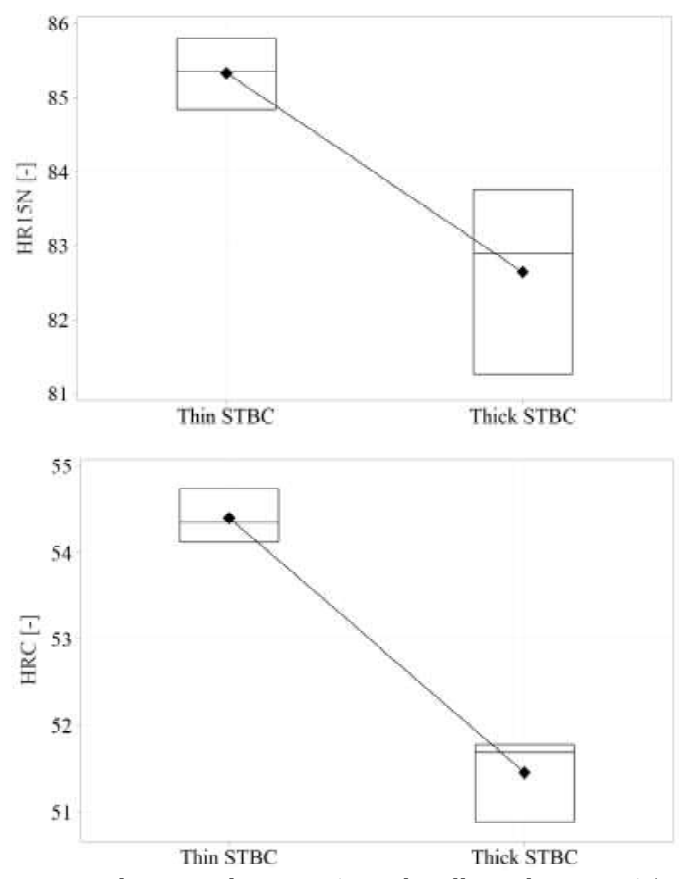

Figure 7: Vickers Hardness HV1, Rockwell Hardness HR15N and HRC in dependence of coating thickness

The results in Fig. 7 show a lower hardness for the thicker STBC samples. Although the difference is small, the macrohardness values of thicker TBCs are clearly lower than those of the thinner ones. The difference is less obvious in microhardness measurements. This is because macrohardness measurement encompasses a larger area of TBC giving an average value of hardness which encompasses the contribution not only the dense segments between vertical cracks but also the cracks and other defects. Microhardness, on the other hand only measures the hardness of the dense segments between vertical cracks and covers smaller areas and hence is less suitable to identify differences in coating microstructures on a larger length scale.

The microstructures of thin and thick STBC samples are shown in Fig. 8. Both samples have an average crack density of about 1.5 vertical cracks per millimeter whose length exceed more than $50 \%$ of the total layer thickness. Both coatings show a porosity of less than $5 \%$. A kind of delamination crack is visible at the interface of the thick STBC which might lead to premature spallation of the coating. This effect is not seen in the thin ceramic layer. According to Benjamin Krebs, this effect of delamination is caused by the different expansion coefficients of the substrate and the coating, the modulus of elasticity and the influence of the layer thickness, resulting in tensile residual stresses in the layer which can then lead to a stress crack [16].

In addition, the vertical crack structures of the thick specimen show numerous connected horizontal cracks. This feature is observed also in the thin STBC microstructure image but to a significantly lesser extent. The cross comparisons of the two different thick samples 

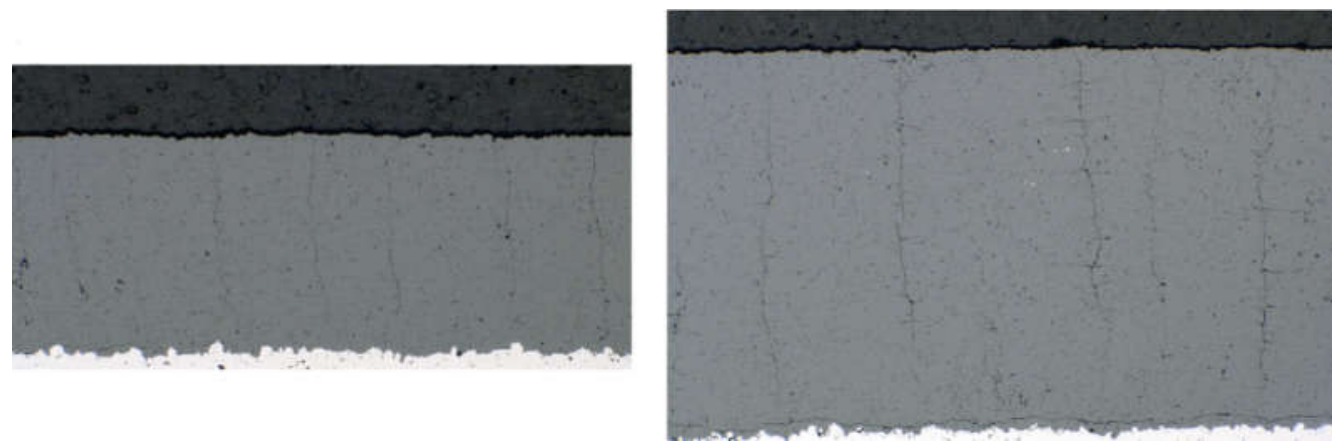

Figure 8: Microsections as-sprayed condition - left: thin STBC; right: thick STBC (same magnification)
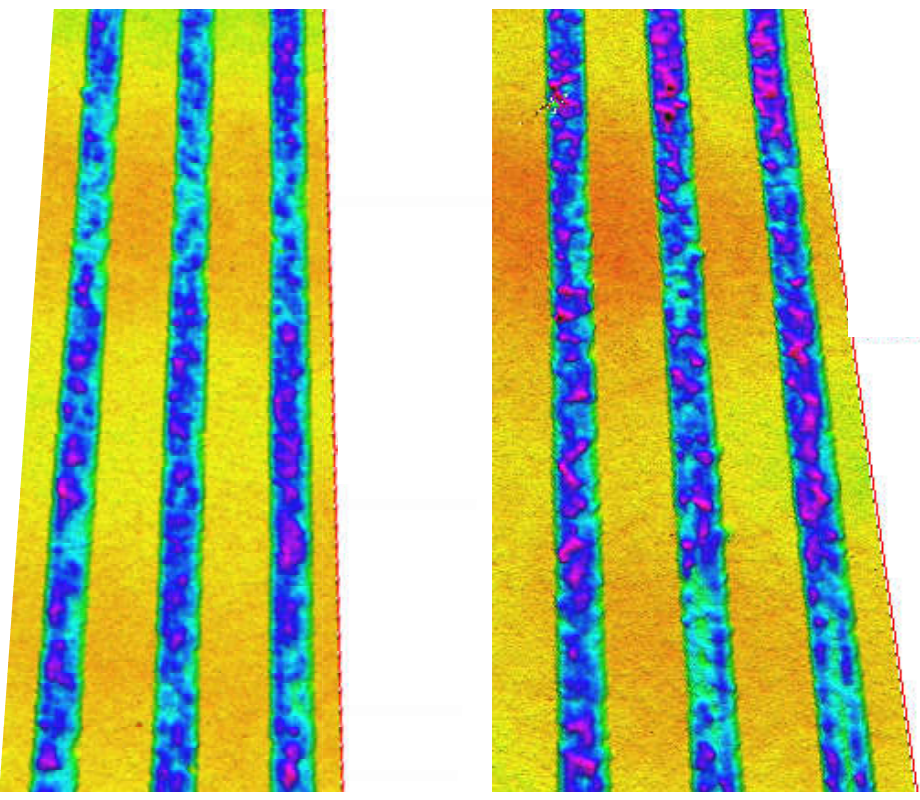

$50-$
$0-$
$-50-$
$-100-$
$-150-$
$-200-$
$-250-$
$-300-$
$-350-$
$-400-$
$-450-$
$-500-$

Figure 9: Optical 3D surface measurements of waterjet machined areas with parameter combination 1 - left: thin STBC; right: thick STBC
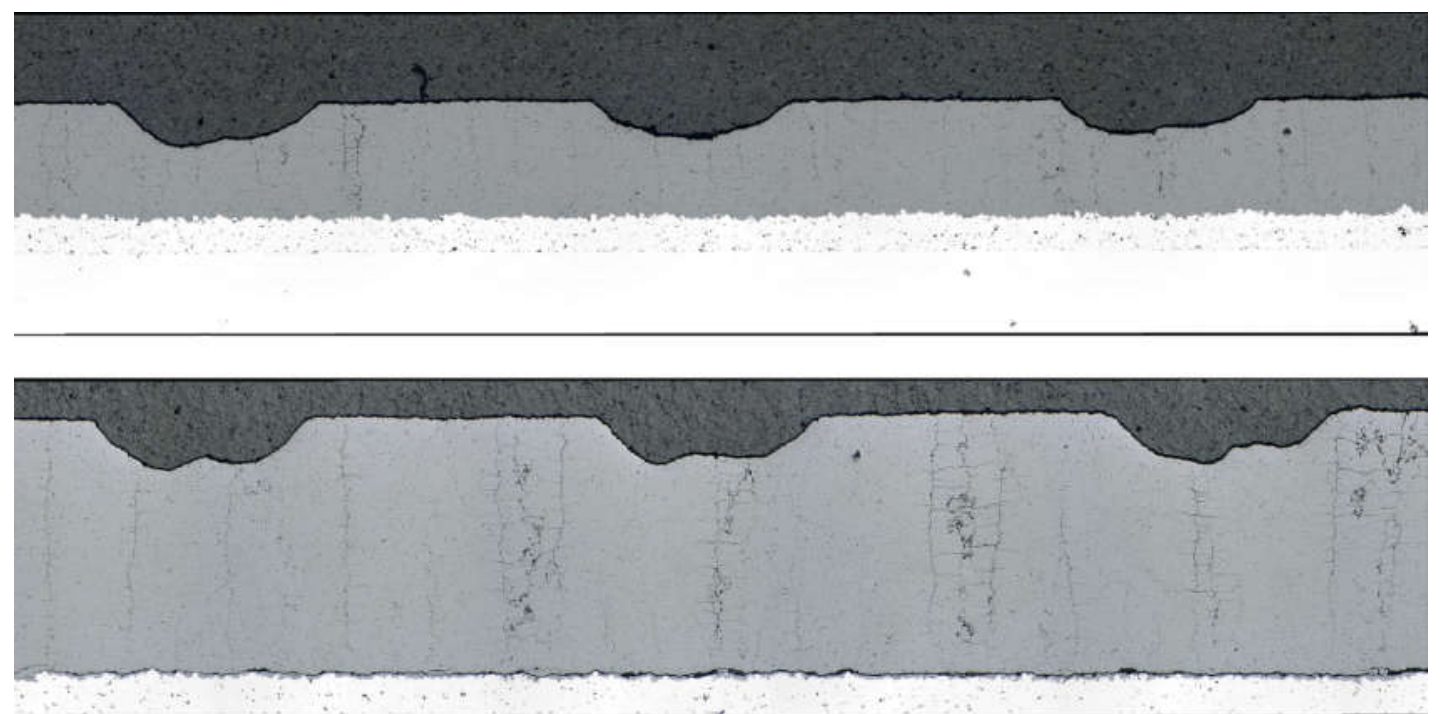

Figure 10: Microsections of waterjet machined areas with parameter combination 1 -

top: thin STBC; bottom: thick STBC (same magnification) 
show that the vertical cracks of the thicker coated samples are more pronounced and opened in their shapes than in the thin STBC samples.

\section{Results of Abrasive Waterjet Machining}

One of the characteristic values after abrasive waterjet machining is the depth of cut of the notches. The depth of cut was measured by the optical 3D surface measurements. Examples of such measurements are shown in Fig. 9. For every notch the depth of cut was measured at four locations. The metallographic cross sections of thin and thick TBC samples after waterjet machining using parameter combination 1 are shown in Fig. 10. It can be seen here that the average depth of cut for thicker coating is larger than that for thinner coating. It is also obvious from the microstructures that there is significantly more horizontal cracking and other defects (crack networking and globular pores) between vertical cracks compared to the thicker coating.

The parameter combination 1 results in a smaller depth of cut than parameter combination 2 which is independent of STBC thickness. Since the abrasive mass flow and pressure is higher in parameter combination 2, a larger number and more accelerated abrasive particles are available in the abrasive waterjet. When these abrasive particles of higher kinetic energy hit the STBC, it leads to an increased depth of cut.

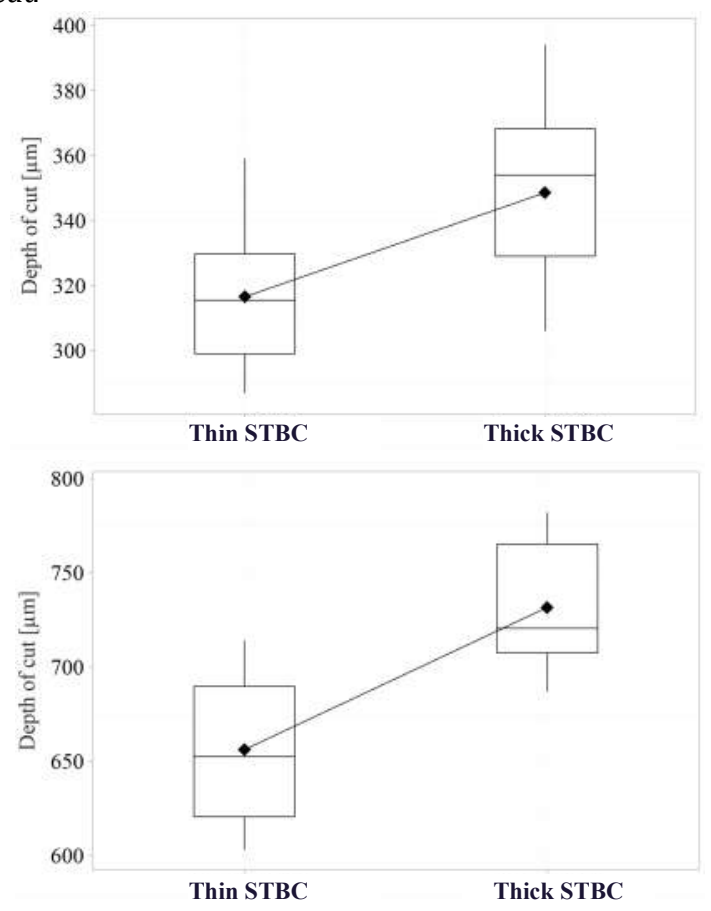

Figure 11: Depth of cut in dependence of coating thickness - top: Parameter combination 1; bottom: Parameter combination 2

The comparison of the depths of cut for the same parameter combination but different STBC thickness is shown in Fig. 11 as box plots. Using the same parameter combination, the mean depth of cut is smaller in case of the thin coating compared to the thick one. This tendency is visible for both parameter combinations. Performing a twosample t-test, it turns out to be significant at a 0.05 level that the mean of depth of cut is smaller in case of the thin coating compared to the thick one. Comparing the standard deviations with each other using another two-sample t-test, there is not enough evidence to conclude that there is a difference in the standard deviation of thin and thick STBCs at a 0.05 level of significance. These findings reveal that there is a thickness-dependent difference in the behavior of the same STBCs when exposed to the abrasive waterjet machining environment wherein thicker coating exhibits lower abrasive/erosive wear resistance.

\section{Discussion}

The results of the previous sections demonstrate that there is a thickness-dependent difference in the microstructure and properties of the STBCs. A study by Nguyen and Wang about the erosion mechanisms in abrasive waterjet machining of brittle materials showed that material hardness, fracture toughness and elastic modulus were the key influencing factors [17]. In the present study hardness was seen to decrease with increasing coating thickness. Shinde et al. [1] showed that the average elastic modulus of the STBC decreases with increasing thickness. These thickness-dependent changes in the properties of STBCs can explain the observed difference in the waterjet machining response of the thin and thick coatings. On a microstructure level, the more pronounced vertical cracks and the presence of higher level of horizontal cracks and other defects between dense segments in thicker coating may be the key contributors to the observed decrease in the hardness and abrasive wear resistance. The following hypothesis is proposed by the authors to explain this microstructural change. As the thickness of dense areas continues to increase to a certain value, the deposition stresses continue to build up. The stresses built up in the coating must be released upon reaching certain thresholds. The first level of stress relief occurs at the onset of macrosegmentation as described by Shinde et al. [1]. The onset of macro-segmentation causes a step decrease in the coating stress at that instant. However, upon further increasing the coating thickness, additional stresses are generated. These stresses are relieved by secondary stress relief mechanisms. The crack opening starts to become larger and more pronounced and an increased amount of horizontal cracking is observed in the microstructure. Occasionally, a crack network with random orientations and even some globular pores may form in some segments and in some cases extreme layering and local debonding can also occur. These secondary stress-relief mechanisms more than compensate for the stresses generated by increasing thickness, resulting in a continuous decrease in the stress with increasing thickness thereby preserving the overall integrity of the coating.

\section{Conclusions}

This paper presented the microstructures and properties of 8YSZ STBCs as a function of coating thickness. With increasing coating thickness, the following trends were observed:

- decrease of hardness values

- more pronounced and opened vertical cracks

- increase of the appearance of short-arm horizontal incipient cracks $\rightarrow$ tendency to layering

- higher potential of interface delamination $\rightarrow$ higher risk of spallation

With a growing industry trend towards thicker STBCs, it is important to understand the microstructure and property changes associated with thickness. The assumption that properties of a STBC applied using a qualified spray parameter remain independent of coating thicknesses may 
lead to significant issues and potential premature failure of parts in the engine. Understanding of the behaviour of coating with respect to their thickness can also help adapt the spray parameters to potentially reduce the coating defects including delamination at the bond-coat or other interfaces. Waterjet machining has been shown to be a promising manufacturing route to carve 3D-features in some gas turbine components to enhance their functionality. It was shown in this paper that waterjet machining response of STBCs is also sensitive to TBC thickness and must be considered during design and process development. In future work, the dependence of STBCs fracture toughness on its thickness and its effect on waterjet machining and other coating characteristics will be investigated.

\section{Acknowledgements}

Research is funded by the German Federal Ministry of Economic Affairs and Energy by resolution of the German Federal Parliament under the funding code 03ET7085. Responsibility for the contents of this publication rests with the authors.

\section{References}

1. Shinde S V, Gildersleeve E J, Johnson C A, Sampath S, Segmentation crack formation dynamics during air plasma spraying of zirconia, Acta Materialia, 2020, 183, 196-206.

2. Sporer D, Girgulis J, Dambra C, and Dorfman M, Segmented 8\% YSZ thermal barrier coating solutions using cascaded arc gun technology, Application noteTP-0002.3 - Segmented 8\% YSZ TBC Coatings - 2016.01, Oerlikon Metco. (2018).

3. Liu D, Flewitt P, Raman measurements of stress in films and coatings, Spectroscopic Properties of Inorganic and Organometallic Compounds, Royal Society of Chemistry, 2014, 45, 141-177.

4. Sharma A, Dudykevych T, Sansom D, Subramanian R, Increased Reliability of Gas Turbine Components by Robust Coatings Manufacturing, Journal of Thermal Spray Technology, 2017, 26, 1084-1094.

5. Herman H, Sampath S, McCune R, Thermal spray: Current status and future trends, MRS Bull., 2000, 25 (07) 17-25.

6. Sampath S, Schulz U, Jarligo M O, Kuroda S, Processing science of advanced thermal-barrier systems, MRS Bull. 2012, 37 (10), 903-910.

7. Evans A G, Mumm D, Hutchinson J, Meier G, Pettit F Mechanisms control- ling the durability of thermal barrier coatings, Prog. Mater Sci., 2001, 46 (5), 505-553.

8. Taylor $\mathrm{T} A$, Thermal barrier coating for substrates and process for producing it, Patent Number US 5073433A. 1989.

9. Guo H B, Murakami H, Kuroda S, Effect of hollow spherical powder size distribution on porosity and segmentation cracks in thermal barrier coatings, J. Am. Ceram. Soc., 2006, 89 (12), 3797-3804.

10. Yang G J, Li C X, Hao S, Xing Y Z, Yang E J, Li C J, Critical bonding temperature for the splat bonding formation during plasma spraying of ceramic materials, Surf. Coat. Technol., 2013, 235, 841-847.

11. Guo H B, Vaßen R, StöverD, Atmospheric plasma sprayed thick thermal barrier coatings with high segmentation crack density, Surf. Coat. Technol., 2004, 186 (3), 353-363.

12. Guo H B, Kuroda S, Murakami H, Segmented thermal barrier coatings produced by atmospheric plasma spraying hollow powders, Thin Solid Films, 2006, 506-507, 136-139.

13. Guo H, Kuroda S, Murakami H, Microstructures and properties of plasma-sprayed segmented thermal barrier coatings, J. Am. Ceram. Soc., 2006, 89 (4), 1432-1439.

14. Karger M, Vaßen R, Stöver D, Atmospheric plasma sprayed barrier coatings with high segmentation crack densities:
Spraying process, microstructure and thermal cycling behavior, Surface and Coatings Technology, 2011, 206(1), 16-23.

15. Dwivedi G, Nakamura T, Sampath S, Determination of thermal spray coating property with curvature measurements, Journal of Thermal Spray Technology, 2013, 22(8), 1337-1347.

16. Krebs B. Konturgenaue Bauteilbeschichtung für den Verschleißschutz mittels Atmosphärischen Plasmaspritzens und Lichtbogenspritzens. Dissertation, Technische Universität Dortmund. Dortmund; 2011.

17. Nguyen T, Wang J, A review on theerosionmechanisms in abrasive waterjetmicromachining of brittlematerials, International Journal of Extreme Manufacturing, 2019, 1, 012006. 